\title{
Magnetoresistance quantum oscillations in a magnetic two-dimensional electron gas
}

\author{
J. Kunc, ${ }^{1,2,{ }^{*}}$ B. A. Piot, ${ }^{1}$ D. K. Maude, ${ }^{1}$ M. Potemski, ${ }^{1}$ R. Grill, ${ }^{2}$ C. Betthausen, ${ }^{3}$ D. Weiss,${ }^{3}$ V. Kolkovsky, ${ }^{4}$ G. Karczewski, ${ }^{4}$ \\ and T. Wojtowicz ${ }^{4}$ \\ ${ }^{1}$ Laboratoire National des Champs Magnétiques Intenses (LNCMI), CNRS-UJF-UPS-INSA, 38042 Grenoble, France \\ ${ }^{2}$ Institute of Physics, Faculty of Mathematics and Physics, 12116 Prague, Czech Republic \\ ${ }^{3}$ Experimentelle und Angewandte Physik, Universität Regensburg, D-93040 Regensburg, Germany \\ ${ }^{4}$ Institute of Physics, Polish Academy of Sciences, 02-668 Warsaw, Poland
}

(Received 10 June 2015; published 12 August 2015)

\begin{abstract}
Magnetotransport measurements of Shubnikov-de Haas ( $\mathrm{SdH})$ oscillations have been performed on twodimensional electron gases (2DEGs) confined in CdTe and CdMnTe quantum wells. The quantum oscillations in $\mathrm{CdMnTe}$, where the 2DEG interacts with magnetic Mn ions, can be described by incorporating the electron-Mn exchange interaction into the traditional Lifshitz-Kosevich formalism. The modified spin splitting leads to characteristic beating pattern in the SdH oscillations, the study of which indicates the formation of Mn clusters resulting in direct anti-ferromagnetic Mn-Mn interaction. The Landau-level broadening in this system shows a peculiar decrease with increasing temperature, which could be related to statistical fluctuations of the Mn concentration.
\end{abstract}

DOI: 10.1103/PhysRevB.92.085304

PACS number(s): 72.80.Ey, 73.21.Fg, 73.63.-b

\section{INTRODUCTION}

At low temperatures, the longitudinal resistivity of metallic systems exhibits quantum oscillations when submitted to a sufficiently high magnetic field. These so-called Shubnikov-de Haas $(\mathrm{SdH})$ oscillations are, in particular, characteristic of two-dimensional electron gases (2DEGs) confined in semiconducting structures and appear at low magnetic fields prior to the development of the quantum Hall effect in clean systems. The analysis of the magnetic field and temperature dependence of the SdH oscillations [1-4] provides valuable information on the quantized density of states (e.g., the Landau-level shape [3,5] and the cyclotron and spin gaps [6]) as well as on the nature of the carrier scattering $[3,4]$ and the associated quantum life times [7,8]. While the most detailed studies were historically undertaken in high-mobility GaAs-based 2DEGs, other 2D systems of high quality have slowly emerged, enabling us to explore the influence of different parameters such as the valley [9-11] and spin degrees of freedom [12,13], as well as the effect of magnetism in these systems [14-16].

In this work, we present an investigation of the $\mathrm{SdH}$ oscillations in a high-quality "magnetic 2DEG" in a diluted magnetic semiconductor, CdMnTe [14,15]. CdMnTe is grown by substituting a small fraction of $\mathrm{Cd}$ atoms by $\mathrm{Mn}$ in the original (nonmagnetic) CdTe II-VI semiconductor. The high quality of these systems was recently demonstrated by the observation of the fractional quantum Hall effect $[12,17]$. A systematic comparison of the $\mathrm{SdH}$ oscillations in both systems is made here to identify the particular effects related to the presence of magnetic $\mathrm{Mn}$ ions. The $\mathrm{SdH}$ oscillations in CdTe exhibit a behavior similar to that of the widely studied GaAs-based 2DEG: a field-/temperature-independent Landaulevel broadening characteristic of a long-range scattering mechanism and an exchange-enhanced spin gap leading to spin-split oscillations (a doubling of the frequency) above a critical magnetic field. In CdMnTe, the $\mathrm{SdH}$ oscillations

*kunc@karlov.mff.cuni.cz exhibit an additional beating pattern with nodes where the oscillations have a vanishing amplitude. We show that this behavior is a consequence of the giant Zeeman splitting (GZS) resulting from the $s$ - $d$ exchange interaction between electrons and the $S=5 / 2 \mathrm{Mn}$ spins [15]. The $\mathrm{SdH}$ characteristics can be well described by incorporating the electron-Mn exchange interaction into the traditional Lifshitz-Kosevich formalism [18]. For a good quantitative description, the formation of $\mathrm{Mn}$ pair clusters with direct $\mathrm{Mn}-\mathrm{Mn}$ antiferromagnetic interactions, which reduce the average $\mathrm{Mn}$ spin polarization, has to be considered. Another peculiarity of the magnetic 2DEG is a decrease of the Landau-level broadening with increasing temperature, together with an increase in the broadening with increasing magnetic field. This suggests a connection between the Landau-level broadening and the Mn spin polarization, as expected in the presence of local fluctuation in the $\mathrm{Mn}$ concentration.

\section{SAMPLES}

The nonmagnetic CdTe sample consists of a 20-nm-wide CdTe quantum well $(\mathrm{QW})$ that is modulation doped with iodine on one side and embedded between $\mathrm{Cd}_{0.74} \mathrm{Mg}_{0.26}$ Te barriers. The spatial profiles of the conduction band $(\mathrm{CB})$ and valence band (VB) at the $\Gamma$ point in the direction perpendicular to the QW plane are shown in Fig. 1(a). The band profiles have been calculated solving self-consistently the Schrödinger and Poisson equation including many-body electron-electron exchange interaction within the mean-field approximation. The magnetic sample consists of a 21.1-nm-wide $\mathrm{Cd}_{1-x} \mathrm{Mn}_{x} \mathrm{Te}$ QW. The average Mn concentration of $\sim 0.3 \%$ is introduced by $\delta$-doping within 7 separate monolayers among the 65 CdTe monolayers composing the QW. The spatial profile of manganese doping is shown in Fig. 1(b). Fick's law for manganese diffusion was considered assuming a manganese diffusion length of 1 monolayer of CdTe [19]. The samples, in the form of $1.5 \times 6 \mathrm{~mm}$ rectangles, were fitted with electrical contacts in a Hall barlike configuration. Experiments have been carried out in ${ }^{3} \mathrm{He} /{ }^{4} \mathrm{He}$ dilution refrigerator inserted 


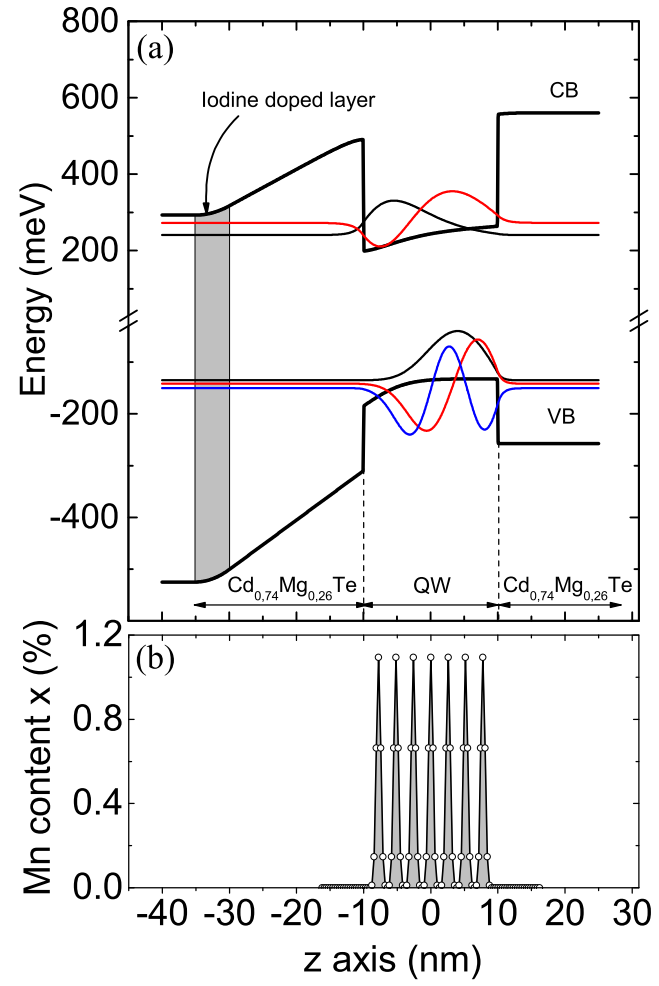

FIG. 1. (Color online) (a) Conduction-band (CB) and valenceband (VB) spatial profiles (perpendicular to the plane of QW) of the CdTe QW based on the self-consistent solution of the Schrödinger and Poisson equation. The position of the iodine $\delta$-doped layer is shown by gray area. The first two (three) electron (heavy-hole) bound states are depicted by black and red (black, red, and blue) curves. (b) The position of the manganese $\delta$-doped layers in CdMnTe QW. The diffusion length of manganese in CdTe was taken into account and set to be one CdTe monolayer. The discrete points are plotted at the positions of CdTe monolayers.

into a superconducting magnet. A standard, low-frequency $(\approx 10 \mathrm{~Hz}$ ) lock-in technique has been applied for the resistance measurements. The samples were illuminated by using the 514-nm line of an $\mathrm{Ar}^{+}$laser to increase the 2DEG mobility by 20\%-30\%. The permanent illumination also increases carrier concentration by $\approx 5 \%$. The laser illumination was limited to $\sim 50 \mu \mathrm{W} / \mathrm{cm}^{2}$ and was permanently maintained as it was found to ensure the most stable conditions over the different experimental runs. The resulting heating effects on the $2 \mathrm{DEG}$ were estimated directly from the magnetoresistance. Special attention has been paid to using slow sweeps so as not to affect the amplitude of fast SdH oscillations. The field-sweeping speed for the CdTe QW was $10 \mathrm{mT} / \mathrm{min}$ for $B=0-0.3 \mathrm{~T}$ and $15 \mathrm{mT} / \mathrm{min}$ for $B=0.3-1 \mathrm{~T}$, and it was $15 \mathrm{mT} / \mathrm{min}$ in the whole range of fields $B=0-1 \mathrm{~T}$ for the CdMnTe QW. Under our experimental conditions, the CdTe 2DEG density was $4.5 \times 10^{11} \mathrm{~cm}^{-2}$ (corresponding to a Fermi energy of $E_{F}=$ $10.8 \mathrm{meV}$ ), with a low-temperature mobility of $\mu=2.6 \times$ $10^{5} \mathrm{~cm}^{2} / \mathrm{Vs}$. The CdMnTe 2DEG density was $4.0 \times 10^{11} \mathrm{~cm}^{-2}$ (corresponding to a Fermi energy of $E_{F}=9.6 \mathrm{meV}$ ), with a low-temperature mobility of $\mu=1.2 \times 10^{5} \mathrm{~cm}^{2} / \mathrm{Vs}$. The lower carrier density in the CdMnTe QW is caused by the

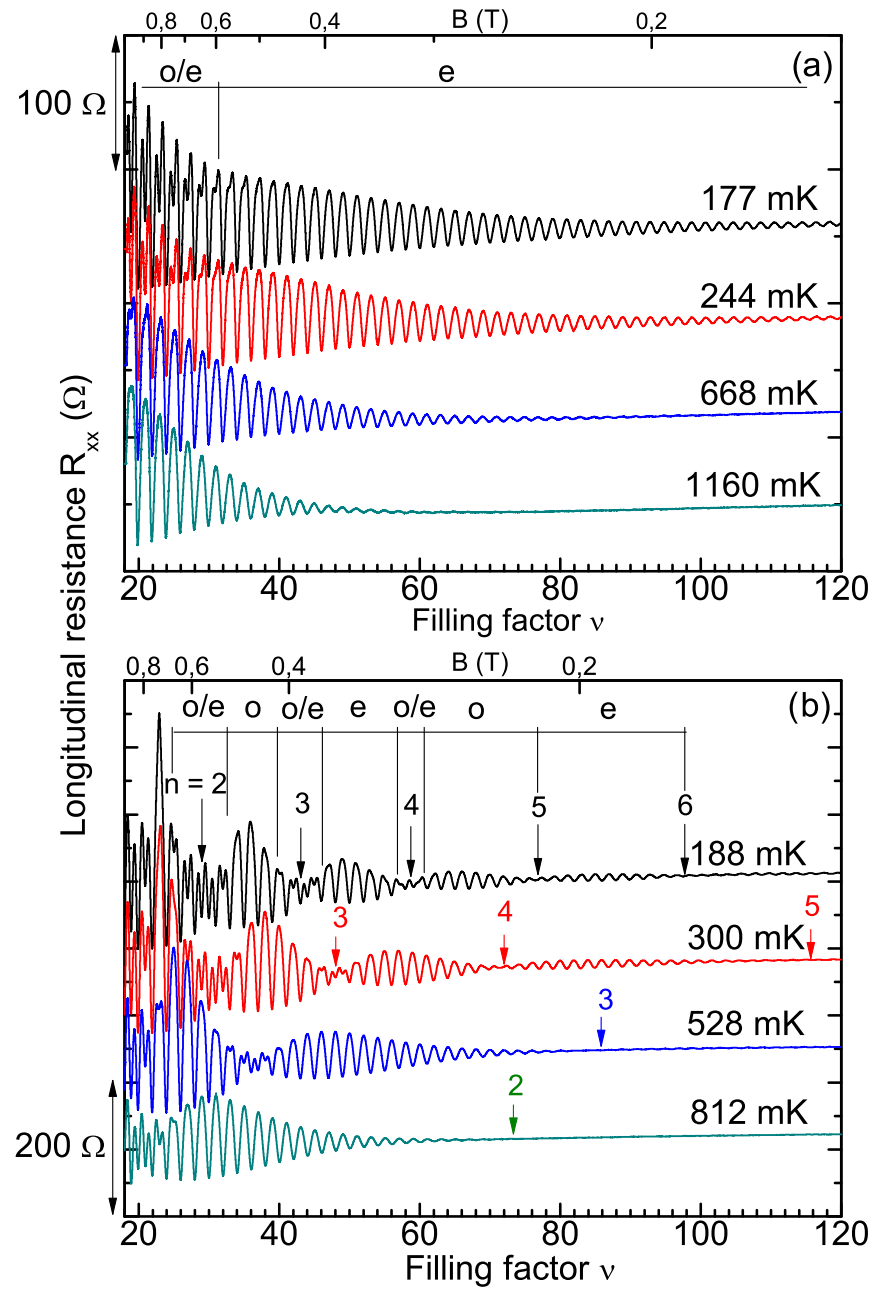

FIG. 2. (Color online) Longitudinal magnetoresistance in (a) CdTe and (b) CdMnTe QWs for four selected temperatures. The data are shifted along the $y$ axis for clarity, and the units of (a) $100 \Omega$ and (b) $200 \Omega$ are marked along the left side by a double arrow. Minima corresponding to odd, even, and alternating odd and even filling factors are labeled "o," "e," and "o/e," respectively. The nodes [condition $\Delta_{s}=(n+1 / 2) \hbar \omega_{c}$ ] in the beating pattern of magnetoresistance of CdMnTe QW are labeled by integer index $n$.

1.1-nm-wider well and different band gap of CdMnTe. Both vary eigenenergies in the $\mathrm{QW}$ and hence also the carrier density, which is given by the alignment of the iodine donor levels and the electron ground state.

The effective mass and the $g$ factor of electrons in CdTe, $m_{e}=0.1 m_{0}$ and $\left|g_{e}\right|=1.6$, were determined by far-infrared magnetoabsorption and Raman scattering spectroscopy.

\section{EXPERIMENTAL RESULTS}

The magnetoresistance of the 2DEG in CdTe and CdMnTe QWs is shown for four selected temperatures in Figs. 2(a) and 2(b), respectively. For the sake of comparison we plot the data as a function of the filling factor $v=B_{1} / B$, where $B_{1}$ is the magnetic field at filling factor $v=1\left(B_{1}=18.8 \mathrm{~T}\right.$ in the CdTe QW and $B_{1}=16.5 \mathrm{~T}$ in the CdMnTe QW). The magnetoresistance in the CdTe QW exhibits the well-known 
SdH oscillations, whose amplitude increases (decreases) with magnetic field (temperature). At low magnetic fields, before spin splitting is observed, the minima of the longitudinal resistance $R_{x x}$ correspond to the situations where the Fermi energy lies between two Landau levels (LL) in a minimum of the total density of states $G_{\text {tot }}$. When the Fermi energy lies in the center of a Landau level, maxima in $R_{x x}$ are observed. Above a critical magnetic field, electron-electron exchange interactions lift the Landau-level spin degeneracy [6], which leads to alternating odd- and even-filling-factor minima in the SdH oscillations [visible, e.g., for $v<30$ at $T=177 \mathrm{mK}$ in Fig. 2(a)].

The magnetoresistance in the CdMnTe QW also exhibits $\mathrm{SdH}$ oscillations, as can be seen in Fig. 2(b). However, an additional beating pattern is observed, and "nodes" can be distinguished in the $\mathrm{SdH}$ amplitude, as previously observed in Ref. [15]. At low magnetic fields, the $\mathrm{SdH}$ amplitude tends to zero in the region of the nodes, while at higher fields, they are characterized by a local minimum of the $\mathrm{SdH}$ amplitude associated with a doubled $\mathrm{SdH}$ oscillation frequency. The presence of a strong electron-manganese exchange interaction gives rise to a GZS in the 2DEG, which grows quickly as the localized Mn spins are polarized by the applied magnetic field. The GZS saturates when the Mn spin polarization has reached its maximum value for a magnetic field of typically $\sim 0.5 \mathrm{~T}$ at low temperatures. This "Brillouin-like" strong field dependence of the GZS, compared to the smaller linear increase of the cyclotron gap $\left(\hbar \omega_{c}=1.16 \mathrm{meV} / \mathrm{T}\right)$, leads to the rather unusual situation where the spin gap $\Delta_{s}$ can be several times larger than the cyclotron gap. As the magnetic field increases, the conditions $\Delta_{s}=n \hbar \omega_{c}$, where $n$ is a (decreasing) integer, are successively satisfied. This magnetic-field-dependent commensurability of the spin and cyclotron gaps leads to a maximized density of states when the Fermi level lies in the center of coinciding (degenerate) levels. When the spin-resolved Landau levels are all equally spaced $\left[\Delta_{s}=(n+1 / 2) \hbar \omega_{c}\right]$, the maximum density of states is a factor of 2 smaller. The observed $\mathrm{SdH}$ beating is therefore a direct manifestation of the GZS in magnetic 2DEGs. We note that the node conditions $\left[\Delta_{s}=(n+1 / 2) \hbar \omega_{c}\right]$ should also be accompanied by a doubling of the $\mathrm{SdH}$ frequency, similar to the one observed at high enough magnetic fields in CdTe [Fig. 2(a)]. This is indeed observed for small $n$, as indicated by the down arrows in Fig. 2(b). In the lower magnetic field regime, where $\hbar \omega_{c} \leqslant \Gamma$, the density-of-states modulation is so small that the nodes conditions result in a disappearance of the $\mathrm{SdH}$.

The fast Fourier transform (FFT) of the data for both nonmagnetic and magnetic QWs is shown in Fig. 3. The FFT was performed in the range of magnetic fields $B=0.1-0.54$ $\mathrm{T}$ in order to take into account the low-field oscillations where the spin-resolved Landau levels can be neglected. The nonmagnetic QW and the magnetic QW at $812 \mathrm{mK}$ show single-frequency resistance oscillations in $1 / B$, as expected from the data in Fig. 2. The low-temperature $\mathrm{SdH}$ oscillations in a magnetic QW show double-frequency oscillations, and the distance between both frequencies decreases at elevated temperature. The frequency difference is proportional to the GZS, as explained within the model proposed in the following section.
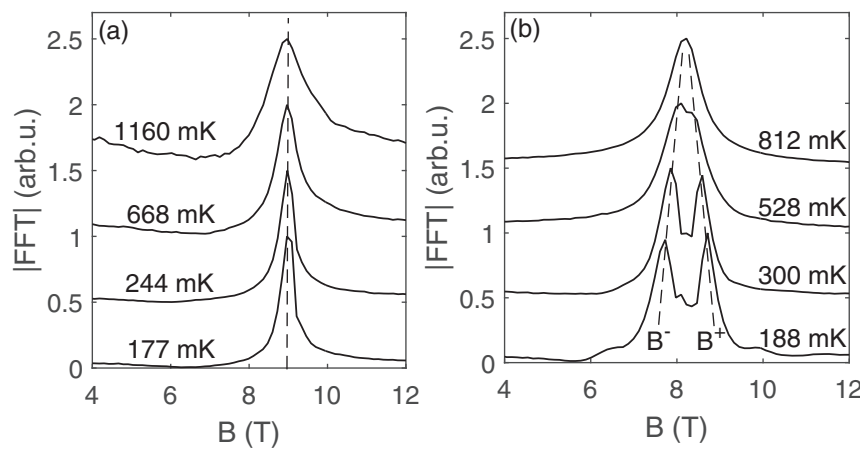

FIG. 3. Amplitude of the fast Fourier transform (FFT) of the data shown in Fig. 2. The Fourier transform of the SdH oscillations in (a) CdTe and (b) CdMnTe QWs. The FFT was performed on data in the low-field interval $B=0.1-0.54 \mathrm{~T}$, where effects of spin-resolved Landau levels can be neglected.

\section{THEORETICAL MODEL}

In order to quantitatively describe the data in both CdTe and $\mathrm{CdMnTe}$, we have derived the formula describing the $\mathrm{SdH}$ oscillation in the case of an arbitrarily large spin splitting $\Delta_{s}$. We have used the Kubo-Greenwood expression $[8,20]$

$$
\sigma(B)=\int_{-\infty}^{\infty} \sigma(E)\left(-\frac{\partial n_{F D}}{\partial E}\right) d E
$$

to calculate the conductivity $\sigma(B)$ of electrons, where $n_{F D}$ is the Fermi-Dirac distribution. The conductivity $\sigma(E)$ calculated within the Drude model yields $\sigma(E)=e^{2} n_{\mathrm{eff}} / m_{e} \tau_{\mathrm{tr}} \omega_{c}^{2}$ in the diffusion limit $\left(\omega_{c} \tau_{\mathrm{tr}} \gg 1\right)$, where $n_{\text {eff }}$ is an effective carrier concentration contributing to $\sigma(E), m_{e}$ is the effective mass of electrons, $\tau_{\text {tr }}$ is the transport lifetime, and $\omega_{c}$ is the cyclotron frequency.

The effective carrier concentration $n_{\text {eff }}$ is proportional to the density of states at the Fermi level $G\left(E_{F}\right)$ and can be written as $n_{\text {eff }} \propto \frac{G\left(E_{F}\right)}{G_{0}}=1+\frac{\delta G\left(E_{F}\right)}{G_{0}}$, where $G_{0}=\frac{m_{e}}{2 \pi \hbar^{2}}$ is the zero-field density of states and $\delta G(E)$ is the modulation of $G(E)$ for $B>0 \mathrm{~T}\left[G(E)=G_{0}+\delta G(E)\right.$ and $\left.\delta G(E) \ll G_{0}\right]$.

The relative change of the conductivity can then be written as $\left|\frac{\sigma_{x x}(B)-\sigma_{0}}{\sigma_{0}}\right|=2^{p}\left|\frac{\delta G}{G_{0}}\right|$, where the exponent $p$ depends on the type of scattering ( $p=1$ for long-range scattering and $p=2$ for short-range scattering where $\left.1 / \tau_{\mathrm{tr}} \propto \frac{G\left(E_{F}\right)}{G_{0}}[21]\right)$. $G(E)$ has been modeled as a sum of either Lorentzian [Eq. (2)] or Gaussian [Eq. (3)] Landau levels:

$$
\begin{gathered}
G(E)=\frac{m_{e}}{2 \pi \hbar^{2}} \frac{\hbar \omega_{c}}{\pi \Gamma} \sum_{n=0}^{\infty} \sum_{s= \pm 1 / 2} \frac{1}{1+\left(\frac{E-E_{n, s}}{\Gamma}\right)^{2}}, \\
G(E)=\frac{m_{e}}{2 \pi \hbar^{2}} \frac{\hbar \omega_{c}}{\sqrt{2 \pi} \Gamma} \sum_{n=0}^{\infty} \sum_{s= \pm 1 / 2} \exp \left[-\frac{\left(E-E_{n, s}\right)^{2}}{2 \Gamma^{2}}\right],
\end{gathered}
$$

where $\Gamma$ is the half width at half maximum (HWHM) of the Landau levels ( $\Gamma=\hbar / 2 \tau_{q}$, where $\tau_{q}$ is the quantum lifetime) and $E_{n, s}$ is the energy of the LL with orbital (spin) quantum number $n(s= \pm 1 / 2)$. In order to compare the model directly with the resistance data, we have used the relation $\left|\frac{\sigma_{x x}(B)-\sigma_{0}}{\sigma_{0}}\right|=$ $\left|\frac{R_{x x}(B)-R_{0}}{R_{0}}\right|$, where $\sigma_{0}$ and $R_{0}$ are the zero-field conductivity and the resistance, respectively, valid for a $2 \mathrm{DEG}$ in a quantizing magnetic field. Hence, the final expression for the resistance 
reads, in the form of the Fourier series,

$$
\begin{aligned}
& \left|\frac{R_{x x}(B)-R_{0}}{R_{0}}\right| \\
& =2^{p} \sum_{s=1}^{\infty}(-1)^{s} \exp \left[-2\left(\frac{\pi \Gamma s}{\hbar \omega_{c}}\right)^{l}\right] \frac{s 2 \pi^{2} k_{B} T_{e} / \hbar \omega_{c}}{\sinh \left(s 2 \pi^{2} k_{B} T_{e} / \hbar \omega_{c}\right)} \\
& \quad \times \cos \left(\frac{2 \pi E_{F} s}{\hbar \omega_{c}}\right) \cos \left(\frac{\pi s \Delta_{s}}{\hbar \omega_{c}}\right)
\end{aligned}
$$

Equation (4) comprises the case of dominant long-range $(p=1)$ and short-range $(p=2)$ scattering mechanisms, Lorentzian $(l=1)$, and Gaussian $(l=2)$ LL broadening. We note that the LL shape is not unambiguously determined from Eq. (4): a $B$-independent Lorentzian broadening $\Gamma_{L}$ is equivalent to a $B$-dependent $\left(\Gamma_{G} \propto \sqrt{B}\right)$ Gaussian broadening $\Gamma_{G}$, where $\Gamma_{L}=\frac{\pi}{\hbar \omega_{c}} \Gamma_{G}^{2}$. The terms "Lorentzian" and "Gaussian" broadening are used here in the sense of magneticfield-independent broadening.

Finally, an arbitrarily large spin splitting $\Delta_{s}$ is taken into account by the last cosine term [22-24]. In CdTe, at low magnetic fields, the spin splitting is much smaller than the cyclotron energy such that $\cos \left(\frac{\pi s \Delta_{s}}{\hbar \omega_{c}}\right) \approx 1$, which does not influence much the $\mathrm{SdH}$ amplitude. In contrast, in CdMnTe, $\Delta_{s}$ at low magnetic field can be much larger than $\hbar \omega_{c}$, which leads to the beating patterns described in the previous section. In this case, the cosine term in Eq. (4) describes the additional modulation of the envelope of the $\mathrm{SdH}$ oscillations.

We note that in the case of a nonmagnetic CdTe QW the spin splitting is negligible; hence, there is only one dominant cosine term in Eq. (4), giving rise to single-frequency resistance oscillations, as seen in FFT [Fig. 3(a)]. The GZS in a magnetic QW is even larger than the cyclotron gap; hence, both cosine terms in Eq. (4) play an important role. The product of the two cosine terms in Eq. (4) can be rewritten as $\cos \left(\frac{2 \pi E_{F} s}{\hbar \omega_{c}}\right) \cos \left(\frac{\pi s \Delta_{s}}{\hbar \omega_{c}}\right)=$ $\frac{1}{2}\left[\cos \left(\frac{2 \pi E_{F} s}{\hbar \omega_{c}}+\frac{\pi s \Delta_{s}}{\hbar \omega_{c}}\right)+\cos \left(\frac{2 \pi E_{F} s}{\hbar \omega_{c}}-\frac{\pi s \Delta_{s}}{\hbar \omega_{c}}\right)\right]$, giving rise to two frequencies, $B^{ \pm}=\frac{m}{\hbar e}\left(E_{F} \pm \frac{\Delta_{s}}{2}\right)$, as depicted in Fig. 3(b) by dashed lines. The value $\Delta_{S, F F T}=1.4 \mathrm{meV}\left(\Delta_{S, F F T}\right.$ is spin gap $\Delta_{s}$ determined from the FFT peaks at $B^{ \pm}$) at $T=188 \mathrm{mK}$ is the field-averaged value of the spin gap. The range of magnetic fields, over which $\Delta_{S}$ is averaged to obtain $\Delta_{S, F F T}$, is determined by the interval of magnetic fields used to perform numerically FFT ( $B=0.1-0.54 \mathrm{~T})$.

\section{DATA MODELING}

\section{A. SdH oscillations in CdTe}

The amplitude of the $\mathrm{SdH}$ oscillations in CdTe is plotted in Fig. 4 for carrier temperatures from 177 to $1200 \mathrm{mK}$. The amplitudes are compared with the model (4), using a Landau-level broadening $\Gamma=(112 \pm 10) \mu \mathrm{eV}, m_{e}=0.1 m_{0}, R_{0}=65 \Omega$, and $g_{e}=-1.6$. The carrier temperature $T_{e}$, which differs from the bath temperature $T_{\text {bath }}$ in our experimental conditions, was determined as a fitting parameter. $T_{e}$ (used throughout the paper) is plotted versus $T_{\text {bath }}$ in Fig. 8 below. The results were found to be essentially the same when taking into account one or more terms in the Fourier series of Eq. (4). The data are well described by a long-range scattering formalism $(p=1)$ and Lorentzian Landau levels $(l=1)$. The dominant

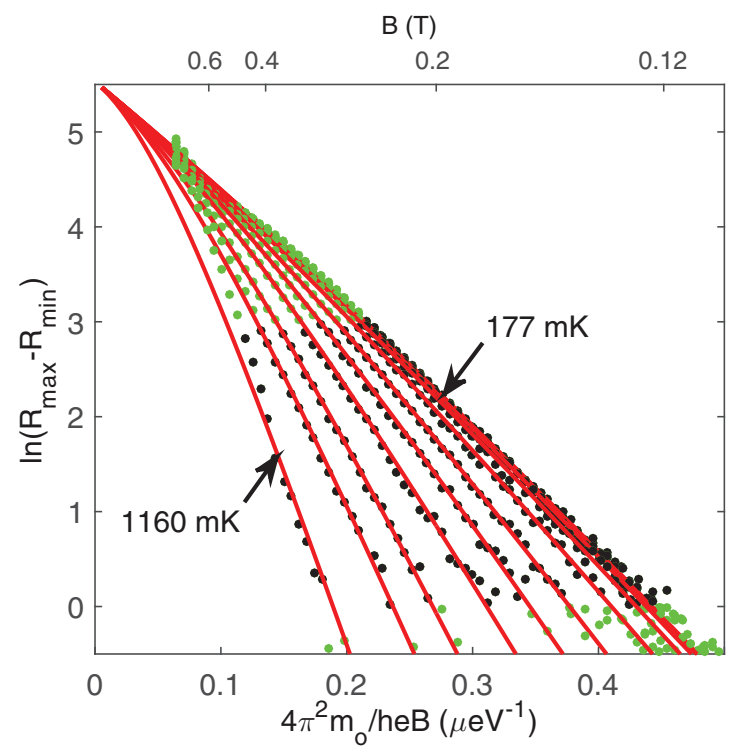

FIG. 4. (Color online) Natural logarithm of the amplitude of $\mathrm{SdH}$ oscillations in CdTe for several carrier temperatures from 177 to $1160 \mathrm{mK}$ (solid circles). Due to the limited range of validity of Eq. (4) and/or the smallness of the signal-to-noise ratio, only the black points were used in the fitting procedure. The red curves are the theoretical fits using Eq. (4) with a Landau-level broadening $\Gamma=110 \mu \mathrm{eV}$ and an effective mass $m_{e}=0.1 m_{0}$.

role of the long-range scattering mechanism, as well as the extracted value for the quantum lifetime $\tau_{q}=(3 \pm 0.3) \mathrm{ps}$, is a fingerprint of a good sample quality, sufficient to observe the integer and fractional quantum Hall effects in this II-VI semiconductor material, as reported in our earlier study [12].

\section{B. SdH oscillations in CdMnTe}

The SdH oscillations in CdMnTe are analyzed in two steps. We first focus on the position of the nodes (Sec. V B 1) and then discuss the oscillation amplitude (Sec. V B 2). The final overall behavior is summarized in Sec. V B 3.

\section{Characteristics of the SdH nodes}

The temperature dependence of the magnetic-field position of the $\mathrm{SdH}$ nodes is shown in Fig. 5. As explained in Sec. III, the nodes appear when the condition $\Delta_{s}=\left(n+\frac{1}{2}\right) \hbar \omega_{c}$ is fulfilled. The spin splitting $\Delta_{s}$ in CdMnTe can be modeled in a meanfield approach as

$$
\begin{aligned}
\Delta_{s}= & g_{e} \mu_{B} B+\Delta_{\operatorname{exch}} \mathcal{B}_{5 / 2}\left[\frac{\frac{5}{2} g_{M n} \mu_{B} B}{k_{B}\left(T_{M n}+T_{0}\right)}\right]\left(1-P_{p}\right) \\
& +\frac{\Delta_{\operatorname{exch}} P_{p}}{2 S_{0}} \sum_{n=1}^{5} \frac{1}{\exp \left(\frac{2 n J_{A F}-g_{M n} \mu_{B} B}{k_{B} T_{M n}}\right)+1}+\alpha \Delta_{s},
\end{aligned}
$$

where the four terms are the bare Zeeman splitting; the GZS due to the $s$ - $d$ exchange interaction between electrons and isolated Mn spins [25]; the contribution of antiferromagnetic (AFM) interactions within pair clusters of Mn atoms [26], which modifies the average Mn spin polarization; and the contribution of electron-electron interactions [27], respectively. 


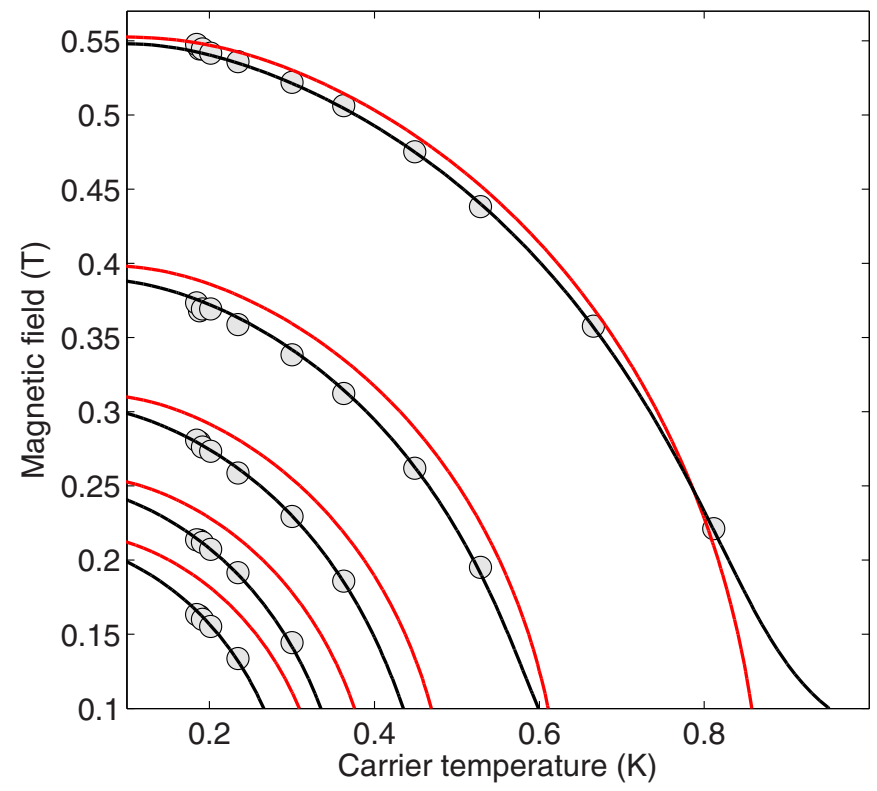

FIG. 5. (Color online) Magnetic field position of the nodes in the beating pattern of $R_{x x}$ in a CdMnTe QW plotted vs carrier temperature. Experimental data (points) are compared with the models of GZS including/neglecting Mn pair clusters (black/red curves). The mean AFM exchange interaction was included by $T_{0}=(40 \pm 10) \mathrm{mK}$ and $T_{0}=(90 \pm 10) \mathrm{mK}$ in the two models of GZS, respectively.

The strength of the $s-d$ electron-manganese interaction $\Delta_{\text {exch }}$ depends in particular on the Mn concentration (nominally, $x_{\text {ave }}=0.3 \%$ ) and the total Mn spin quantum number $S_{0}=$ $5 / 2$. $\mathcal{B}_{5 / 2}$ is the Brillouin function describing the Mn spin magnetism, where $g_{M n}=2.0$ is the manganese $g$ factor. $T_{0}$ is an additional phenomenological temperature which can be introduced to take into account AFM Mn-Mn interactions through the (single-particle) Brillouin function. $T_{M n}$ is $\mathrm{Mn}$ temperature (we note that the best fit to the data was obtained with $T_{M n}=T_{e}$ ). The "cluster" term aims at directly including the effect of AFM Mn-Mn interactions on the spin polarization of the $S_{0}=5 / 2 \mathrm{Mn}$ system. $P_{p}$ is the probability that Mn is a part of a pair cluster, and $J_{A F}$ is the strength of the direct Mn-Mn AFM interaction between two neighbors. $\alpha \cong \frac{\Delta_{0}}{2 E_{F}}=0.11$ stands for the electron-electron interaction (in our case, the Fermi energy is $E_{F}=9.6 \mathrm{meV}$, and the parameter $\Delta_{0}=2.1 \mathrm{meV}$ has been determined in previous work [27]).

We have used two different approaches to fit the experimental data of Fig. 5. Parameters $\Delta_{\text {exch }-1}\left(\Delta_{\text {exch-2 }}\right)$ and $T_{0-1}\left(T_{0-2}\right)$ are used in the following text to denote results of numerical fitting of the parameters $\Delta_{\text {exch }}$ and $T_{0}$ in Eq. (5) within the first (second) approach. In the first approach, the $e$-Mn interaction is entirely taken into account by the second term of Eq. (5), and the third term is neglected $\left(P_{p}=0\right)$. The best fits to the experimental data are reported as red curves in Fig. 5 and describe the data at the qualitative level. The corresponding parameters are $\Delta_{\text {exch-1 }}=1.7 \mathrm{meV}$ and $T_{0-1}=90 \mathrm{mK}$, which phenomenologically take into account the $\mathrm{Mn}-\mathrm{Mn}$ interaction by reducing the average $\mathrm{Mn}$ spin polarization.
In the second approach, the Mn-Mn AFM interaction within pair clusters is directly taken into account by using the third (cluster) term of Eq. (5). In this term, the nearestneighbor (NN) AFM interaction is expected to be rather strong $\left(J_{N N} / k_{B} \approx 5 \mathrm{~K}[25,26]\right)$, such that for magnetic fields lower than $5 \mathrm{~T}\left[=\left(2 J_{N N}-5 k_{B} T_{e, \max }\right) /\left(g_{M n} \mu_{B}\right)\right.$, where $\left.T_{e, \max }=823 \mathrm{mK}\right], \mathrm{NN}$ are always antiferromagnetically coupled and thus do not contribute to the GZS. However, the next-nearest neighbors (NNN) of manganese atom interaction are weaker $\left(J_{N N N} / k_{B}=0.5 \mathrm{~K}\right.$ [28-31]) and can play a role already at $B \sim 0.5 \mathrm{~T}$ and $T \sim 200 \mathrm{mK}$ and similar or lower $B / T$ values. We note that the interaction strength between third- and higher-order $\mathrm{NN}$ is generally small and decreases exponentially with distance $[31,32]$. Their residual influence is sufficiently well described by the commonly used $T_{0}$ phenomenological parameter [25]. Besides these distant pairs, AFM interactions from higher-order clusters (triplets, quadruplets, etc.) are also included in $T_{0}$. The fits to the data obtained in this second approach are plotted as black curves in Fig. 5 and give an excellent quantitative description of the data. The fitting parameters are $P_{p}=20 \%, J_{A F} / k_{B}=0.5 \mathrm{~K}$, $\Delta_{\text {exch-2 }}=1.7 \mathrm{meV}$, and $T_{0-2}=(40 \pm 10) \mathrm{mK}$. The extracted probability of cluster formation, $P_{p}=20 \%$, is significantly higher than the one expected from statistical considerations (typically a few percent). This is usually explained in terms of nonhomogeneous distribution of $\mathrm{Mn}$ [33]. The value of $J_{A F} / k_{B}=0.5 \mathrm{~K}$ suggests that the influence of the Mn pair clusters originates from the NNN interaction of manganese ions. The small but nonzero value observed for $T_{0-2}=(40 \pm$ 10) $\mathrm{mK}$ can be attributed to higher-order AFM interactions.

In conclusion, the beating pattern of the $\mathrm{SdH}$ oscillations is profoundly modified by the magnetic subsystem and therefore constitutes a powerful tool to characterize the $e$-Mn interaction, the Mn concentration, and the Mn-Mn interactions in CdMnTe systems.

\section{Oscillation amplitude}

In Fig. 6, we plot the $\mathrm{SdH}$ oscillation amplitude in our CdMnTe QW for two representative temperatures, using a reciprocal magnetic field scale. In addition to the previously analyzed beating patterns, which manifest themselves as repeated deviations from the solid black line, we observe that the overall envelope of the amplitude of the oscillations depends only weakly on temperature. This is particularly evident when comparing the almost parallel solid black lines in Fig. 6 to the case of CdTe (Fig. 4), where the 1/B slope of the oscillation amplitude strongly increases with temperature, a usual consequence of the $\mathrm{SdH}$ temperature damping. This suggests that the usual $\mathrm{SdH}$ temperature damping, described by the $x / \sin (x)$ function in Eq. (4), is compensated by some nontrivial temperature dependence of the disorder damping [the exponential term in Eq. (4)]. More precisely, the observed behavior points toward an unusual temperature narrowing of the LL broadening (at thus a reduced "disorder damping" at higher temperature) which cannot be anticipated within the most standard forms of scattering. Another interesting observation is the nonlinearity of the overall envelope in the $1 / B$ scale, which is indicated at low temperature by the red 


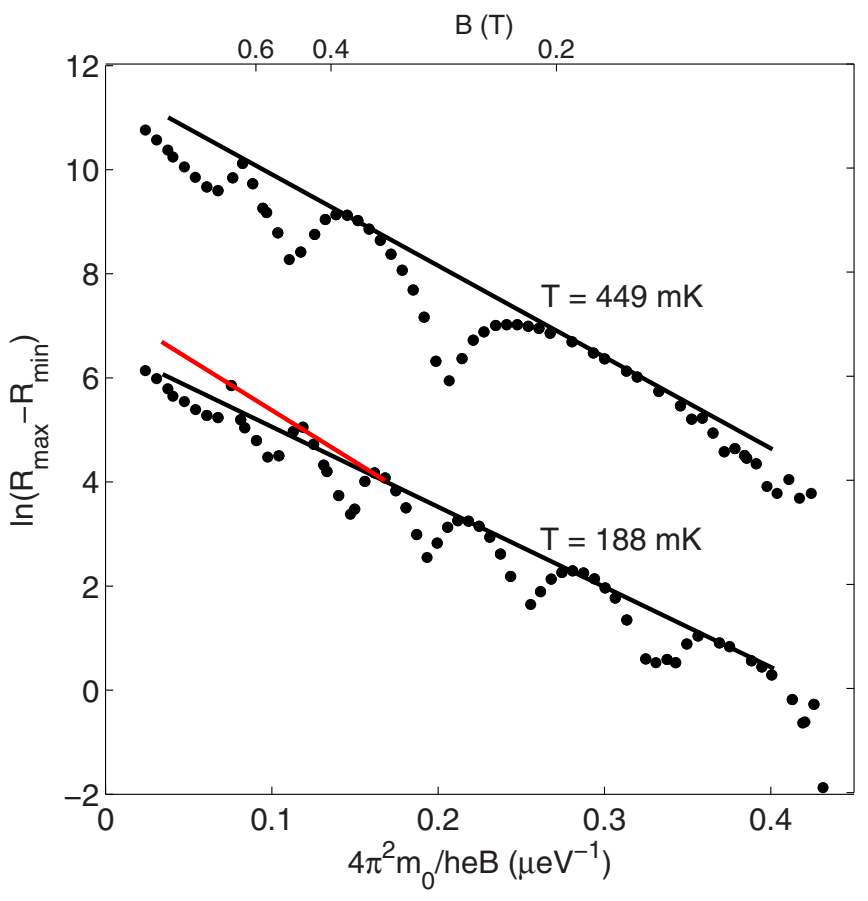

FIG. 6. (Color online) Natural logarithm of SdH oscillation amplitude in CdMnTe at the electron temperatures 188 and $449 \mathrm{mK}$ in reciprocal magnetic field scale. The two straight black lines show the usual linear $1 / B$ behavior expected within the LifshitzKosevich formalism, nevertheless exhibiting an unusual parallelism for different temperatures. The red line depicts nonlinearity, described in our model as a field-dependent Landau-level broadening. The data are shifted vertically for clarity.

line in Fig. 6. This $1 / B$ nonlinearity implies a magnetic field dependence of the Landau-level broadening $\Gamma$.

We note that the values of quantum lifetimes in CdTe and $\mathrm{CdMnTe}$ are here very similar, in agreement with previous observations [12,17]. However, the observed field and temperature dependence of this quantity in CdMnTe points to the occurrence of an additional physical effect contributing to the level broadening. Again, the main difference between CdTe and CdMnTe QWs is the presence of manganese spins. As a matter of fact, the observed dependence of the broadening is qualitatively reminiscent of the behavior of the average manganese spin polarization $\left\langle S_{z}\right\rangle$, which increases (decreases) with the magnetic field (temperature), as can be seen by considering the (dominant) Brillouin function in Eq. (5). As the spin polarization of the manganese system directly determines the position of the Landau level, a variation of the manganese content $x$ which appears in the prefactor $\Delta_{\text {exch }}$ in Eq. (5) will shift the energy-level position proportionally to $\left\langle S_{z}\right\rangle$. A nonhomogeneous Mn distribution at the local scale is therefore at the origin of an additional level broadening. The mean energy shift $\Gamma_{M n}$ can be simply written as

$$
\Gamma_{M n}=\left(\frac{\Delta x}{x_{\text {ave }}}\right)\left(\frac{1}{2} \Delta_{\text {exch }}\right) \mathcal{B}_{5 / 2}\left[\frac{\frac{5}{2} g_{M n} \mu_{B} B}{k_{B}\left(T_{M n}+T_{0}\right)}\right],
$$

where $\Delta x$ represents the maximum Mn spatial fluctuation around the average value $x_{\text {ave }}$ (the extremal values of $x$ are then $x=x_{\text {ave }} \pm \Delta x$ ). The other parameters are as defined

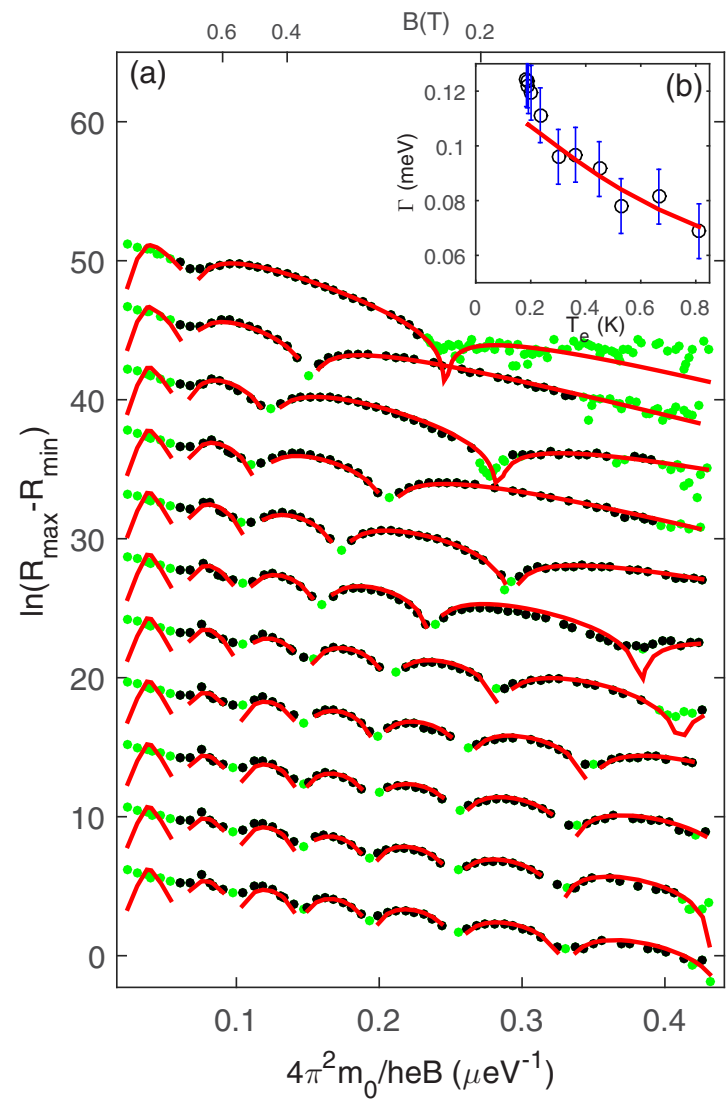

FIG. 7. (Color online) (a) Natural logarithm of the amplitude of $\mathrm{SdH}$ oscillations in a CdMnTe QW for electron temperatures from 188 to $823 \mathrm{mK}$. Experimental data used (unused) in the fitting procedure are plotted by black (green) points, and the red curves show the theoretical model including fluctuations of the manganese concentration as an additional source of Landau-level broadening. (b) Resulting total LL broadening $\Gamma$ as a function of the carrier temperature $T$. The $B$ dependence originating from Eq. (6) is averaged in order to get solely the temperature dependence (red line).

previously, and the mean energy shift $\Gamma_{M n}$ can be identified with the Lorentzian HWHM used in our formalism (2) and (4).

We have reproduced our experimental data by taking this effect into account and writing the total LL broadening in CdMnTe as $\Gamma=\Gamma_{0}+\Gamma_{M n}$, where $\Gamma_{0}$ is a temperature-/fieldindependent broadening and $\Gamma_{M n}$ is the "fluctuation-induced" contribution described above. This broadening was directly injected in the previously used $\mathrm{SdH}$ formalism [Eq. (4)]. The amplitude of SdH oscillations is shown in Fig. 7(a) together with simulations using a LL broadening $\Gamma=\Gamma_{0}+\Gamma_{M n}$ (red solid curves).

As in the case of CdTe, we have used Lorentzian Landau levels in a long-range scattering approximation $[p=1$ in Eq. (4)]. The data were fitted using $\Gamma_{0}=20 \mu \mathrm{eV}$ and $\frac{\delta x}{x_{\text {we }}}=11 \% \pm 5 \%$, which gives an estimation for the relative mean fluctuation of the Mn concentration. This value is in good agreement with the expected statistical fluctuation of the number of manganese ions $N_{M n / e}$ per one electron (or per area defined by the de Broglie wavelength). In our sample $N_{M n / e} \approx 60$, giving fluctuations $\frac{1}{\sqrt{N_{M n / e}}}=13 \%$. 


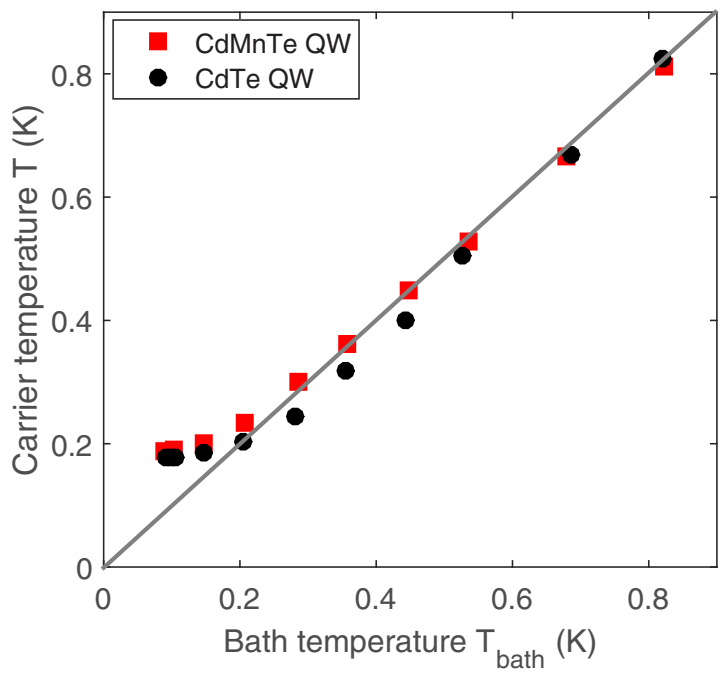

FIG. 8. (Color online) Relation between carrier temperature $T$ and bath temperature $T_{\text {bath }}$ in CdTe (black dots) and CdMnTe (red squares) QWs.

The resulting total LL broadening $\Gamma$ is plotted as a function of magnetic field and temperature in Fig. 7(b). The extracted "nonmagnetic" broadening $\Gamma_{0}$ is smaller in CdMnTe than in CdTe. This is actually not surprising because our approach considers an additional source of broadening in CdMnTe, while the total broadening is similar in both systems. Physically, a smaller $\Gamma_{0}$ broadening in CdMnTe could be attributed to a reduction of the intra-Landau-level spin-flip scattering in CdMnTe. Indeed, while the opposite spin levels in CdTe always belong to the same Landau level, in CdMnTe the GZS puts into coincidence opposite spins with different orbital quantum numbers, which might affect the spin-flip scattering processes. We finally note that the value of $\frac{\delta x}{x_{x}}=11 \% \pm 5 \%$ is obtained by assuming that the temperature-/field-dependent Landau-level broadening originates only from fluctuation in the manganese concentration and thus constitutes an upper bound for the mean fluctuations. Other mechanisms involving the manganese spin polarization $\left\langle S_{z}\right\rangle$, such as an anisotropic electron-Mn interaction similar to the Mn-Mn anisotropic Dzyaloshinskii-Moriya interaction [34], could also contribute to the observed broadening.

The carrier temperature, being the second fitting parameter, is compared for both nonmagnetic and magnetic QWs in Fig. 8. In spite of the lowest bath temperature of $90 \mathrm{mK}$, the carrier temperature saturates at 177 and $188 \mathrm{mK}$ in nonmagnetic and magnetic QWs, respectively. The saturation is probably caused by the $\mathrm{cw}$ laser illumination. However, further experiments need to be done to confirm this conclusion.

\section{Overall behavior}

In Fig. 9, we report the experimental data of Fig. 2(b), together with the model developed throughout the paper for the temperature and field dependence of the $\mathrm{SdH}$ oscillation in CdMnTe. The oscillation amplitude and the node position are well reproduced, as shown earlier in the paper. The beating pattern and the phase shift of the oscillations across each node are correctly described by the last cosine term in Eq. (4)

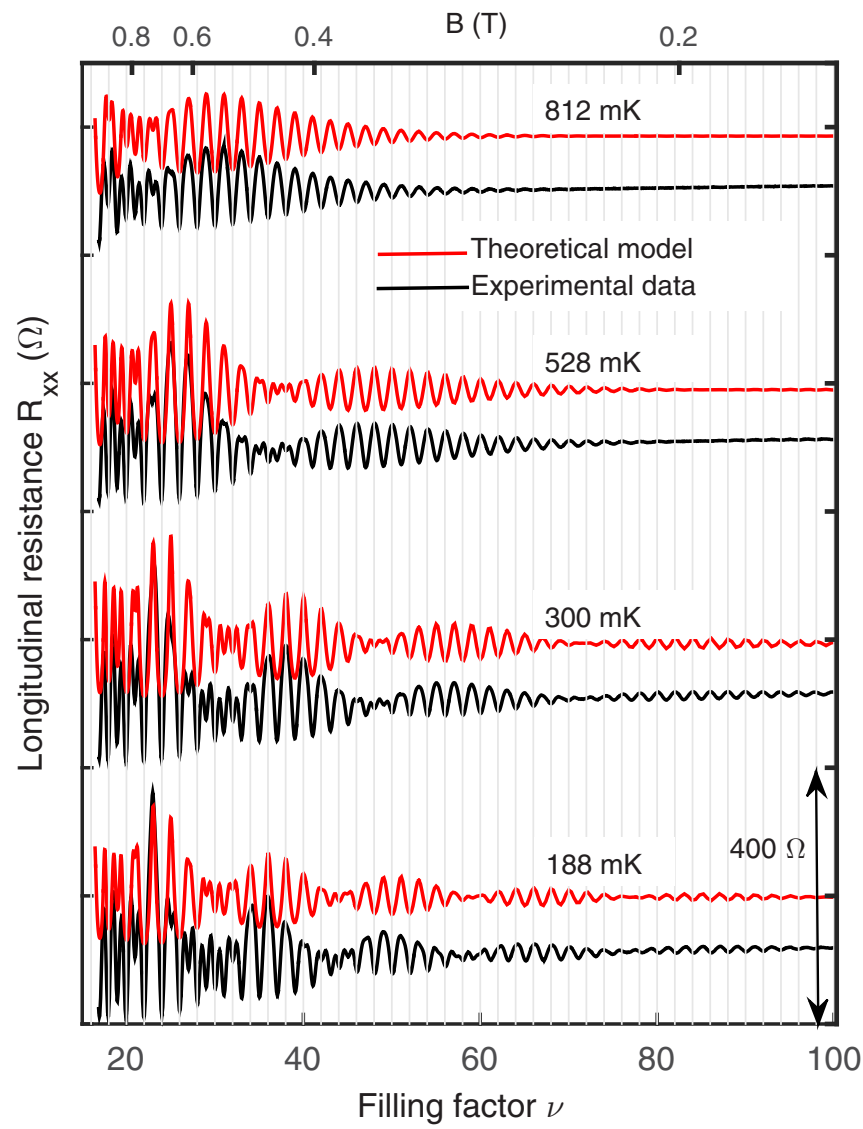

FIG. 9. (Color online) Longitudinal resistance $R_{x x}$ in the CdMnTe QW (black curves) compared with our model (red curves) assuming manganese pair clusters and fluctuations of the manganese concentration. The theoretical model is shifted vertically from the experimental data for clarity. Sets of theoretical model and experimental data for different temperatures are also shifted vertically for clarity.

including the giant Zeeman splitting. We note that in order to reproduce the doubled oscillation frequency at the high-field nodes $(v<50)$, the first two terms of the Fourier series in Eq. (4) have to be taken into account. Taking into account higher-order terms (up to 100) deepens the splitting of the doubled $\mathrm{SdH}$ oscillations, which is nevertheless still weaker than in our experimental observations. This could be related to the proximity of the Stoner transition, where spin splitting develops in a nonlinear way [6,35].

\section{CONCLUSIONS}

Shubnikov-de Haas oscillations have been studied in a high-quality magnetic 2DEG formed in a diluted magnetic CdMnTe quantum well. The $\mathrm{SdH}$ characteristics can be well described by incorporating the electron-Mn exchange interaction in the traditional Lifshitz-Kosevich formalism in a mean-field approach. A more detailed analysis reveals the role of antiferromagnetic Mn-Mn interactions in this system, as well as a nontrivial reduction of the Landau-level broadening with increasing temperature, which can be accounted for by fluctuations in the manganese concentration. 


\section{ACKNOWLEDGMENTS}

We thank P. Kossacki, M. Goryca, and M. Orlita for helpful discussions. This work was part of the research program
MSM0021620834 financed by the Ministry of Education, Youth and Sports of the Czech Republic. The research in Poland was partially supported by National Science Centre (Poland) Grant No. DEC-2012/06/A/ST3/00247.
[1] P. N. Argyres and E. N. Adams, Phys. Rev. 104, 900 (1956).

[2] E. M. Lifshits and A. M. Kosevich, J. Phys. Chem. Solids 4, 1 (1958).

[3] T. Ando, J. Phys. Soc. Jpn. 37, 1233 (1974).

[4] A. Isihara and L. Smrčka, J. Phys. C 19, 6777 (1986).

[5] A. Endo and Y. Iye, J. Phys. Soc. Jpn. 77, 064713 (2008).

[6] B. A. Piot, D. K. Maude, M. Henini, Z. R. Wasilewski, K. J. Friedland, R. Hey, K. H. Ploog, A. I. Toropov, R. Airey, and G. Hill, Phys. Rev. B 72, 245325 (2005).

[7] P. T. Coleridge, P. Zawadzki, and A. S. Sachrajda, Phys. Rev. B 49, 10798 (1994).

[8] P. T. Coleridge, H. Hayne, P. Zawadzki, and A. S. Sachrajda, Surf. Sci. 361/362, 560 (1996).

[9] K. v. Klitzing, G. Dorda, and M. Pepper, Phys. Rev. Lett. 45, 494 (1980).

[10] K. Takashina, M. Brun, T. Ota, D. K. Maude, A. Fujiwara, Y. Ono, Y. Takahashi, and Y. Hirayama, Phys. Rev. Lett. 99, 036803 (2007).

[11] K. S. Novoselov, A. K. Geim, S. V. Morozov, D. Jiang, Y. Zhang, S. V. Dubonos, I. V. Grigorieva, and A. A. Firsov, Science 306, 666 (2004).

[12] B. A. Piot, J. Kunc, M. Potemski, D. K. Maude, C. Betthausen, A. Vogl, D. Weiss, G. Karczewski, and T. Wojtowicz, Phys. Rev. B 82, 081307 (2010).

[13] A. Tsukazaki, A. Ohtomo, T. Kita, Y. Ohno, H. Ohno, and M. Kawasaki, Science 315, 1388 (2007).

[14] S. Scholl, H. Schafer, A. Waag, D. Hommel, K. von Schierstedt, B. Kuhn-Heinrich, and G. Landwehr, Appl. Phys. Lett. 62, 3010 (1993).

[15] F. J. Teran, M. Potemski, D. K. Maude, T. Andrearczyk, J. Jaroszynski, and G. Karczewski, Phys. Rev. Lett. 88, 186803 (2002).

[16] H. Buhmann, E. G. Novik, V. Daumer, J. Liu, Y. S. Gui, C. R. Becker, and L. W. Molenkamp, Appl. Phys. Lett. 86, 212104 (2005).

[17] C. Betthausen, P. Giudici, A. Iankilevitch, C. Preis, V. Kolkovsky, M. Wiater, G. Karczewski, B. A. Piot, J. Kunc, M. Potemski et al., Phys. Rev. B 90, 115302 (2014).
[18] I. M. Lifshitz and A. M. Kosevich, Sov. Phys. JETP 2, 636 (1956).

[19] P. Kossacki, N. T. Khoi, and J. A. Gaj, Superlattices Microstruct. 16, 63 (1994)

[20] R. M. Hill, J. Phys. C 9, L381 (1976).

[21] P. T. Coleridge, R. Stoner, and R. Fletcher, Phys. Rev. B 39, 1120 (1989).

[22] F. F. Fang and P. J. Stiles, Phys. Rev. 174, 823 (1968).

[23] A. E. Stephens, D. G. Seiler, J. R. Sybert, and H. J. Mackey, Phys. Rev. B 11, 4999 (1975).

[24] E. V. Kurganova, H. J. van Elferen, A. McCollam, L. A Ponomarenko, K. S. Novoselov, A. Veligura, B. J. van Wees, J. C. Maan, and U. Zeitler, Phys. Rev. B 84, 121407 (2011).

[25] J. A. Gaj, R. Planel, and G. Fishman, Solid State Commun. 29 , 435 (1979).

[26] R. L. Aggarwal, S. N. Jasperson, P. Becla, and R. R. Galazka, Phys. Rev. B 32, 5132 (1985).

[27] J. Kunc, K. Kowalik, F. J. Teran, P. Plochocka, B. A. Piot, D. K. Maude, M. Potemski, V. Kolkovsky, G. Karczewski, and T. Wojtowicz, Phys. Rev. B 82, 115438 (2010).

[28] M. M. Kreitman, F. J. Milford, R. P. Kenan, and J. G. Daunt, Phys. Rev. 144, 367 (1966).

[29] G. Bastard and C. Lewiner, Phys. Rev. B 20, 4256 (1979).

[30] Y. Shapira, S. Foner, D. H. Ridgley, K. Dwight, and A. Wold, Phys. Rev. B 30, 4021 (1984).

[31] M. A. Novak, O. G. Symko, D. J. Zheng, and S. Oseroff, J. Appl. Phys. 57, 3418 (1985).

[32] B. E. Larson, K. C. Hass, H. Ehrenreich, and A. E. Carlsson, Phys. Rev. B 37, 4137 (1988).

[33] R. R. Galazka, S. Nagata, and P. H. Keesom, Phys. Rev. B 22 , 3344 (1980).

[34] N. Samarth and J. K. Furdyna, Phys. Rev. B 37, 9227 (1988).

[35] B. A. Piot, D. K. Maude, M. Henini, Z. R. Wasilewski, J. A. Gupta, K. J. Friedland, R. Hey, K. H. Ploog, U. Gennser, A. Cavanna et al., Phys. Rev. B 75, 155332 (2007). 